\title{
Determination of the IGRF 2000 model
}

\author{
Nils Olsen ${ }^{1}$, Terence J. Sabaka ${ }^{2}$, and Lars Tøffner-Clausen ${ }^{3}$ \\ ${ }^{1}$ Danish Space Research Institute, Juliane Maries Vej 30, DK - 2100 Copenhagen $\emptyset$, Denmark \\ ${ }^{2}$ Raytheon ITSS, Lanham, MD 20706, U.S.A. \\ ${ }^{3}$ Ørsted Science Data Center, DMI, Lyngbyvej 100, DK - 2100 Copenhagen Ø, Denmark
}

(Received February 25, 2000; Revised July 7, 2000; Accepted July 12, 2000)

\begin{abstract}
The IGRF 2000 has been estimated from magnetic measurements taken by the Ørsted sattelite in summer 1999 . For this purpose, three models have been derived: The first two models were estimated using a few geomagnetic quiet days in May and September 1999, respectively. The third model, called Oersted(10c/99), was derived from scalar data spanning six months and vector data spanning four months. In order to get a model for epoch 2000.0, the IGRF 95 secular variaion model has been applied to the data. The IGRF 2000 model was taken to be the internal degree/order 10 portion of Oersted(10c/99). We describe the data selection, model parameterization, parameter estimation and an evaluation of the three models.
\end{abstract}

\section{Introduction}

Twenty years after the Magsat mission, the Ørsted satellite was launched on February 23, 1999 in a near polar orbit with an inclination of $96.5^{\circ}$, a perigee at $638 \mathrm{~km}$ and an apogee at $849 \mathrm{~km}$. The principal aim of the Ørsted mission is to accurately map the Earth's magnetic field as caused by internal sources and to investigate current systems in the ionosphere and magnetosphere. The satellite is equipped with a scalar Overhauser magnetometer (OVH), a tri-axial fluxgate magnetometer (CSC, for Compact Spherical Coil) and a star imager (SIM) which determines the attitude of the satellite with high accuracy. The Overhauser magnetometer is mounted at the top of a $8 \mathrm{~m}$ long boom; the vector magnetometer and the star imager are mounted closely together on an optical bench which is located $2 \mathrm{~m}$ away from the scalar magnetometer and $6 \mathrm{~m}$ away from the satellite body. Satellite position is determined using GPS. The orbital plane is slowly drifting in local time and was approximately in the noon/midnight plane in summer 1999.

As part of the calibration of the Ørsted instruments, the relative rotation between the coordinate system of the vector magnetometer and that of the star imager has to be determined with an accuracy of a few arcseconds. This is done in-flight by estimating the three Euler angles (which describe the rotation) simultaneously with a model of the Earth's magnetic field. Such a model is called a "Calibration Model" as opposed for instance to a "Standard Field Model" which is derived from calibrated and aligned data (that means using a fixed set of Euler angles). While one could argue for a refinement in the magnetometer attitude estimates as more sophisticated models of the Earth's magnetic field are employed, experience with Magsat data has shown that the Euler angles estimates are probably fairly robust with respect to analyses more detailed than those presented here. Hence,

Copy right(c) The Society of Geomagnetism and Earth, Planetary and Space Sciences (SGEPSS); The Seismological Society of Japan; The Volcanological Society of Japan; The Geodetic Society of Japan; The Japanese Society for Planetary Sciences. it makes sense to work in the "Standard Field Model" mode if one can since there are far fewer complications. However, the IGRF 2000 model had to be finalized by October 30, 1999, and at that time independent high-precision estimates of the Euler angles were not available. The fact that the IGRF 2000 is derived from a "Calibration Model" probably does not take full advantage of the high-precision of the Ørsted data. However, we believe that the model fully satisfies IGRF standards. (See Olsen et al. (2000) for a description of the "Ørsted Initial Field Model", which was derived in spring 2000 from calibrated and aligned data.)

As part of the IGRF model estimation, three "Calibration models" were derived:

- Oersted(10a/99) is based upon data from the six quiet days May 10-11, May 16-17 and May 21-22, 1999. Average data time of this model is 1999.37 , and so is model epoch.

- Oersted(10b/99) is based upon data from September 23 25,1999 . This was a geomagnetically quiet period according to the $K p$ index, but due to the recovery phase of a previous geomagnetic storm, the $D s t$ index reaches values of $-100 \mathrm{nT}$ during the first hours of September 23. Average data time and model epoch is 1999.73 .

- Oersted(10c/99) is based upon scalar data spanning over six months (March 16, 24; April 15; May 10-11, 1617, 21-22; June 6, 14, 21-22; July 4, 17-20; August 14; September 6, 23-25) and vector data spanning four months (May 10-11, 16-17, 21-22; June 21-22; August 14; September 23-25). Average data time of this model is 1999.45; model epoch is 2000.0. The IGRF 95 secular variation (SV) model (Barton, 1997) has been applied rather than allowing the data to adjust the secular variation.

The first two models describe the geomagnetic field at a specific time instant (May and September 1999, respec- 
tively), and secular variation was neglected when estimating the models since the data span only a few days. However, in May 1999 vector data were only available for a few hours per day (resulting in about 1600 vector triples for the six quiet days used in the model) due to various problems, especially with blinding of the star imager. The three quiet days used in September 1999 yield about 1300 vector triplets, indicating that the percentage of attitude availability has improved since May. Global vector data coverage is far from ideal for both the May and the September data set, and therefore a combined model, the third in the above list, was derived. Due to the large data time span it was necessary to account for the secular variation, and the IGRF 95 secular variation model (Barton, 1997) was applied to all observations to derive a model at epoch 2000.0. Since the data are not allowed to adjust the secular variation, the achieved model misfit not only reflects the fit to the main field, but measures also the validity of the applied secular variation model. Note that the maximum degree and order of the secular variation model is $n_{\max }=8$, and therefore the correction of the Oersted(10c/99) model is only to degree and order 8 .

After evaluation of the three models it was decided to use the third model as the basis for the IGRF 2000; hence the IGRF 2000 model is the internal degree/order 10 subset of the Oersted(10c/99) model. The aim was to estimate Gauss coefficients describing internal (core and crust) sources up to degree and order 10 which are as close as possible to the "true" ones, rather than estimating a degree and order 10 model which best fits the Ørsted observations. A subset of Ørsted scalar data which was not included in the 10c/99 model agrees with that model at the $5 \mathrm{nT}$ level, but only at the $15 \mathrm{nT}$ level with respect to the IGRF 2000. This is mostly due to the external coefficient $q_{1}^{0}$; its magnitude is about $20 \mathrm{nT}$, and it is included in the parent model Oersted(10c/99) but not in the IGRF 2000.

\section{Data Selection and Pre-Processing}

During the first months after launch, the Ørsted satellite provided high-precision attitude information only for less than $20 \%$ of the time. The situation has improved considerably during summer 1999, resulting in vector data for more than $90 \%$ of the time during the last months of 1999 . Opposing this trend, the occurrence of geomagnetic quiet days generally decreased from May to September 1999. As a consequence, data selection was a critical issue for deriving the models, since a compromise has to be found between geomagnetic quietness and accuracy of attitude determination.

Only data during 3-hour periods where $K p \leq 1+$ and for which the previous period had $K p \leq 2$ o have been chosen. To minimize the influence of external field-aligned current systems (which are strongest at polar latitudes and have most influence on the field components perpendicular to the main field), vector data were restricted to dipole latitudes $\lambda_{\text {dip }}$ equatorward of $50^{\circ}$. Only night-side data were used to reduce the disturbing influence of ionospheric currents at middle and low latitudes. The scalar and vector data were decimated such that times of measurement were at least 30 seconds apart.

To reduce the noise in the rotation angle $\kappa$ of the star imager (about 30-60 arcseconds rms, which is about 6 times larger than the rms noise in the two other SIM angles Right Ascension and Declination), a third degree polynomial was fitted to 31 values of $\kappa$ (spanning about 35 seconds), and the value of the polynomial corresponding to the midpoint was chosen.

The CSC magnetometer is a fluxgate instrument which has to be calibrated. This is done by comparing the Overhauser scalar magnetometer measurements, say $F_{\mathrm{OVH}}$, and the field magnitudes computed from the CSC vector magnetometer measurements, say $F_{\mathrm{CSC}}$, which are considered functions of offsets, scale values and non-orthogonalities in the instrument. Estimates of these instrument parameters may be realized by adjusting them so as to minimize the Euclidean norm of the vector of $F_{\mathrm{OVH}}-F_{\mathrm{CSC}}$ residuals over some measurement set. The resulting instrument parameters are then applied to the raw CSC vector measurements rendering a set of calibrated vector measurements in an orthogonal coordinate system (details of this calibration can be found in Olsen et al., 2001). While this calibration can deduce non-orthogonalities in the physical CSC coordinate system, it cannot deduce ab-

Oersted(10a/99)

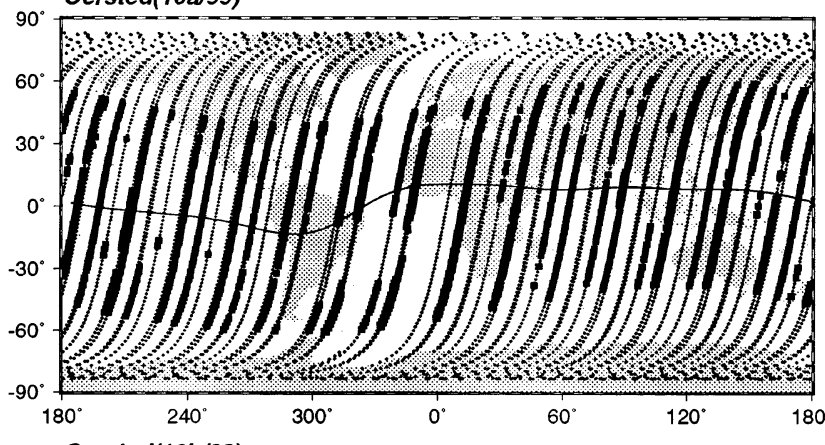

Oersted(10b/99)

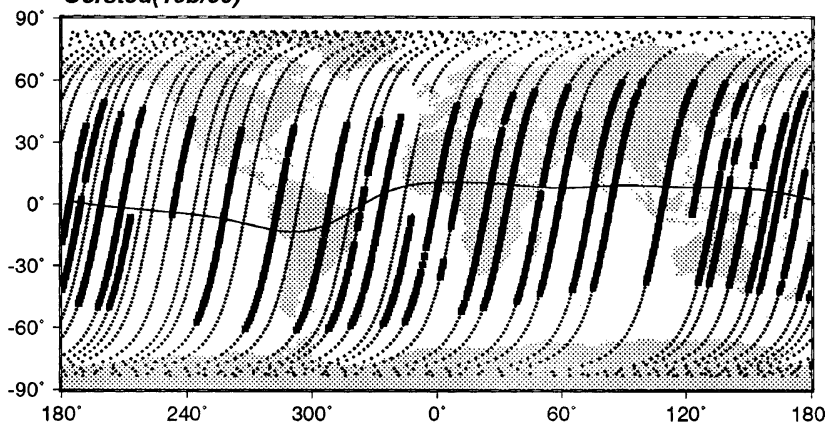

Oersted(10c/99)

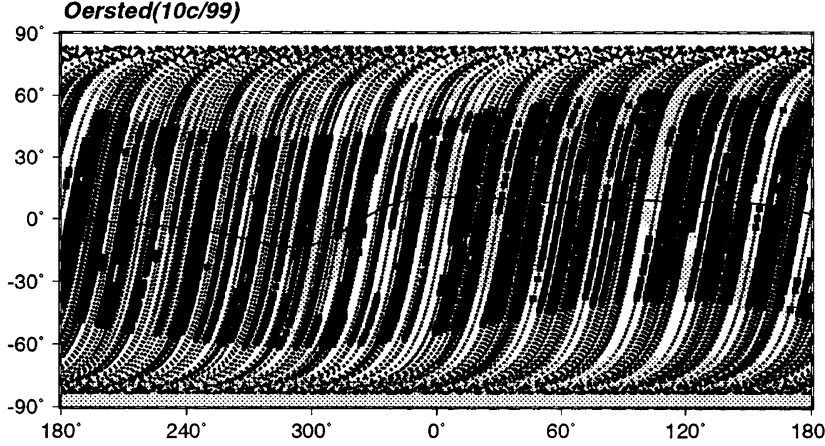

Fig. 1. Distribution of data points used for the three models. Scalar measurements are shown with small symbols and vector measurements with larger symbols. 
solute orientations because only field magnitudes are being compared. Hence, in order to use the calibrated vector data, the rotation from the (known) orthogonal coordinate system of the Ørsted star imager (SIM) to the (unknown) orthogonal coordinate system of the CSC vector magnetometer has to be co-estimated with a field model so that directional information is available. This is part of the modeling scheme reported here, and hence the model consists of two parts: the Gauss coefficients describing the Earth's magnetic field and the Euler angles describing the rotation.

Figure 1 shows the distribution of the data used in deriving the models. Note the lack of vector data over the South Atlantic Anomaly. This is due to incorrect parameter settings of the star imager during the first months of the mission.

\section{Model Parameterization}

Let $\mathbf{T}$ be the rotation matrix which transforms the magnetic field from a local north, east, down $(N E D)$ spherical coordinate system $\mathbf{B}_{N E D}=(N, E, D)^{T}=\left(-B_{\vartheta}, B_{\phi},-B_{r}\right)^{T}$ to the magnetic field $\mathbf{B}_{\mathrm{SIM}}=\underline{\underline{\mathbf{T}}} \cdot \mathbf{B}_{N E D}$ in the coordinate system of the star imager. $\underline{\mathbf{T}}$ is derived from satellite position and from the attitude information given by the star imager. Let $\underline{\underline{\mathbf{R}}}(\boldsymbol{\epsilon})$ be the rotation matrix which transforms from the SIM coordinate system to the orthogonal magnetometer (CSC) coordinate system under the action of a rotation described by the three Euler angles, $\boldsymbol{\epsilon}=(\alpha, \beta, \gamma)^{T}$. Hence the relationship between the magnetic vector in the magnetometer coordinate system and the magnetic vector in the local $N E D$ coordinate system is given by

$$
\begin{aligned}
\mathbf{B}_{\mathrm{CSC}} & =\underline{\underline{\mathbf{R}}}(\boldsymbol{\epsilon}) \cdot \underline{\underline{\mathbf{T}} \cdot \mathbf{B}_{N E D}} \\
& =-\underline{\underline{\mathbf{R}}}(\boldsymbol{\epsilon}) \cdot \underline{\underline{\mathbf{T}}} \cdot \operatorname{grad} V .
\end{aligned}
$$

Here it has been assumed that $\mathbf{B}_{N E D}=-\operatorname{grad} V$ can be derived from a magnetic scalar potential $V$ which is expanded in terms of spherical harmonics:

$$
\begin{aligned}
V= & a\left\{\sum_{n=1}^{N} \sum_{m=0}^{n}\left(g_{n}^{m} \cos m \phi+h_{n}^{m} \sin m \phi\right)\right. \\
& \cdot\left(\frac{a}{r}\right)^{n+1} P_{n}^{m}(\cos \vartheta) \\
& +\sum_{m=0}^{1}\left(q_{1}^{m} \cos m \phi+s_{1}^{m} \sin m \phi\right) \\
& \left.\cdot\left(\frac{r}{a}\right) P_{1}^{m}(\cos \vartheta)\right\} .
\end{aligned}
$$

$a=6371.2 \mathrm{~km}$ is the mean radius of the Earth, $(r, \vartheta, \phi)$ are geographical coordinates, $P_{n}^{m}$ are the associated Schmidt semi-normalized Legendre functions and $\left(g_{n}^{m}, h_{n}^{m}\right)$ and $\left(q_{n}^{m}, s_{n}^{m}\right)$ are the Gauss coefficients describing internal and external sources, respectively. The maximum degree of the spherical harmonic expansion (of internal sources) is chosen as $N=13$. Following the parameterization used for the first Magsat models (cf. the estimation of the GSFC(12/83) model described in Langel and Estes, 1985), the external coefficients and the $g_{1}^{0}$ coefficient are allowed to vary linearly with the Dst index:

$$
g_{1}^{0}=g_{1,0}^{0}+g_{1, D s t}^{0} \cdot D s t
$$

and similarly for $q_{1}^{0}, q_{1}^{1}$ and $s_{1}^{1}$. The number of model parameters is 205 (195 static internal coefficients, 3 static external coefficients, 1 Dst-dependent internal coefficient, 3 Dstdependent external coefficients, and 3 Euler angles). Since the (final) Dst index for the data period was not available at time of modeling, preliminary hourly mean values of Dst as provided by the World Data Center for Geomagnetism, Kyoto (http://swdcdb.kugi.kyoto-u.ac.jp/) have been used.

\section{Estimation of the Model Parameters}

The Gauss coefficients of the field model plus the $3 \mathrm{Eu}-$ ler angles of the rotation matrix $\underline{\underline{\mathbf{R}}}(\boldsymbol{\epsilon})$ were estimated by an iterative Gauss least-squares estimator. The data vector $\mathbf{d}=\left(\mathbf{d}_{\mathrm{OVH}}^{T}, \mathbf{d}_{\mathrm{CSC}}^{T}\right)^{T}$ consists of a first part $\mathbf{d}_{\mathrm{OVH}}=\left(F_{1}, \ldots\right.$, $\left.F_{N_{\mathrm{OVH}}}\right)^{T}$ containing the $N_{\mathrm{OVH}}$ scalar field measurements from the Overhauser instrument, and a second part $\mathbf{d}_{\mathrm{CSC}}=$ $\left(X_{1}, Y_{1}, Z_{1}, \ldots, X_{N_{\mathrm{CSC}}}, Y_{N_{\mathrm{CSC}}}, Z_{N_{\mathrm{CSC}}}\right)^{T}$ containing the $N_{\mathrm{CSC}}$ calibrated vector field measurements from the vector magnetometer in its orthogonal CSC $(X Y Z)$ system. Similarly, the model vector $\mathbf{m}=\left(\mathbf{g}^{T}, \boldsymbol{\epsilon}^{T}\right)^{T}$ consists of a first part $\mathbf{g}=\left(\ldots, g_{n}^{m}, h_{n}^{m}, q_{n}^{m}, s_{n}^{m}, \ldots\right)^{T}$ which contains the Gauss coefficients (and their Dst dependent part) of the spherical harmonic expansion, whereas the second part $\epsilon=(\alpha, \beta, \gamma)^{T}$ contains the three Euler angles describing the "3-2-3" rotation $\underline{\mathbf{R}}(\boldsymbol{\epsilon})$. Note that both the vector (CSC) and the scalar $(\mathrm{OV} \overline{\overline{\mathrm{H}}})$ data are functions of the Gauss coefficients (that is g), but only the CSC measurements are functions of the Euler angles (that is $\boldsymbol{\epsilon}$ ). Therefore, if we denote the data vector predicted from the model, e.g. Eq. (2), with an explicit parameter dependence, such that $\mathbf{d}(\mathbf{m})=\left(\mathbf{d}_{\mathrm{OVH}}^{T}(\mathbf{g}), \mathbf{d}_{\mathrm{CSC}}^{T}(\mathbf{m})\right)^{T}$, then the $i$-th iteration of the Gauss least-squares estimator may be written as

$$
\begin{aligned}
\mathbf{m}_{i+1}= & \mathbf{m}_{i}+\delta \mathbf{m}_{i} \\
\delta \mathbf{m}_{i}= & {\left[\left(\underline{\underline{\mathbf{G}}}_{i}\right)^{T} \cdot \underline{\underline{\mathbf{W}}} \cdot \underline{\underline{\mathbf{G}}}_{i}\right]^{-1} } \\
& \cdot\left[\left(\underline{\underline{\mathbf{G}}}_{i}\right)^{T} \cdot \underline{\underline{\mathbf{W}}} \cdot\left(\mathbf{d}-\mathbf{d}\left(\mathbf{m}_{i}\right)\right)\right]
\end{aligned}
$$

where:

$$
\underline{\underline{\mathbf{G}}}_{i}=\left.\frac{\partial \mathbf{d}(\mathbf{m})}{\partial \mathbf{m}}\right|_{\mathbf{m}=\mathbf{m}_{i}}
$$

and $\underline{\underline{\mathbf{W}}}$ is the data weight matrix, which is diagonal with elements

$$
w_{k k}=\sin \vartheta_{k} / \sigma_{k}^{2},\left\{\begin{array}{l}
\sigma_{k}=5 \mathrm{nT} \text { for OVH scalar data } \\
\sigma_{k}=7 \mathrm{nT} \text { for CSC vector data }
\end{array}\right.
$$

These weighting factors of the form $w \propto \sin \vartheta$ are used to simulate an equal area distribution of the data points on the globe, which is especially crucial when using polar data from a simple equal-time decimation along the orbits of a high-inclination satellite like Ørsted. To account for highlatitude currents, a further latitude dependency of the weights (in addition to the $\sin \vartheta$ weights to simulate an equal area distribution) would be worthwile, but has not been used for deriving the models. Instead, we have tried to reduce their influence by careful data selection. 
Table 1. Number $N_{\text {tot }}$ of data points, number $N_{\text {out }}$ of removed outliers, as well as means and rms misfits (in nT) for the three models.

\begin{tabular}{|c|c|c|c|c|c|c|c|c|c|c|c|c|}
\hline & \multicolumn{4}{|c|}{ Oersted(10a/99) } & \multicolumn{4}{|c|}{ Oersted(10b/99) } & \multicolumn{4}{|c|}{ Oersted(10c/99) } \\
\hline & $N_{t o t}$ & $N_{\text {out }}$ & mean & $\mathrm{rms}$ & $N_{t o t}$ & $N_{\text {out }}$ & mean & $\mathrm{rms}$ & $N_{\text {tot }}$ & $N_{\text {out }}$ & mean & $\mathrm{rms}$ \\
\hline OVH $F$ & 6567 & & -0.01 & 4.40 & 3550 & & 0.13 & 3.06 & 20610 & & 0.04 & 5.21 \\
\hline$\left(\left|\lambda_{\text {dip }}\right|<50^{\circ}\right)$ & & & 0.04 & 2.38 & & & 0.41 & 2.21 & & & 0.13 & 3.29 \\
\hline $\operatorname{CSC} X$ & 1617 & 20 & -1.27 & 3.77 & 1292 & 43 & -1.12 & 3.79 & 4054 & 87 & -1.31 & 4.60 \\
\hline $\operatorname{CsC} Y$ & 1617 & 20 & 0.55 & 6.10 & 1292 & 43 & -0.27 & 6.36 & 4054 & 87 & -0.36 & 6.95 \\
\hline $\operatorname{CSC} Z$ & 1617 & 20 & 1.40 & 5.36 & 1292 & 43 & 0.03 & 5.07 & 4054 & 87 & 1.33 & 5.82 \\
\hline$B_{r}$ & 1617 & 20 & -1.92 & 4.81 & 1292 & 43 & -0.71 & 4.74 & 4054 & 87 & -1.99 & 5.29 \\
\hline$B_{\vartheta}$ & 1617 & 20 & -0.33 & 5.39 & 1292 & 43 & 0.42 & 6.25 & 4054 & 87 & 0.31 & 6.77 \\
\hline$B_{\phi}$ & 1617 & 20 & -0.51 & 5.27 & 1292 & 43 & -0.14 & 4.42 & 4054 & 87 & -0.45 & 5.38 \\
\hline $\operatorname{CSC} B$ & 1617 & 20 & -0.23 & 2.88 & 1292 & 43 & -0.01 & 3.08 & 4054 & 87 & -0.29 & 4.22 \\
\hline $\operatorname{CSC} N$ & 1617 & 20 & 0.73 & 7.74 & 1292 & 43 & -0.14 & 7.53 & 4054 & 87 & 0.36 & 8.14 \\
\hline
\end{tabular}

Three iterations were found to be sufficient when solving the non-linear inverse problem. Outliers were removed before the last iteration; as outlier selection criterion we have used $25 \mathrm{nT}$. If one of the vector residual component was above that value, all three components were removed. We have not removed any scalar data.

\section{Results}

\subsection{Model statistics and data residuals}

Number of data points used, residual means and rms misfits of the three models are given in Table 1.

Note that there is a larger anisotropy of the rms misfit in the CSC coordinate system compared to the $N E D$ coordinate system. This indicates that the cause is not of natural origin (for instance external currents) but due to the instrumentation. The misfit is lowest in CSC $X$, which is the component parallel to the SIM bore-sight. The Star imager parameterizes the measured attitude by three angles: Right Ascension, Declination and Rotation. The first two angles are the pointing of the bore-sight, whereas the third angle is the rotation around the bore-sight axis. The noise in the rotation angle $\kappa$ is about 6 times higher (30-60 arcseconds rms) compared to the first two SIM angles (about 10 arcseconds). This results in a lower rms misfit of the magnetic field component parallel to the SIM bore-sight (that is the CSC $X$ component), as observed. When transformed to the $N E D$ coordinate system, the pronounced noise anisotropy is smeared over all components.

To investigate the noise anisotropy further, the residuals were rotated into a coordinate system where the first axis points along SIM bore-sight (CSC $X$ axis), the second axis is both perpendicular to CSC $X$ and to Earth's magnetic field (this component is called CSC $N$ ), and the third axis completes a right-hand system and is called $\operatorname{CSC} B$, since it is parallel to the projection of the main field onto the CSC $y-z$ plane. As seen in Table 1, almost the whole part of the vector misfit (8.1 nT out of $\sqrt{\mathrm{rms}_{X}^{2}+\mathrm{rms}_{Y}^{2}+\mathrm{rms}_{Z}^{2}}=10.2 \mathrm{nT}$ for the Oersted(10c/99) model) is concentrated in the CSC $N$ component. As described in Section 2, the SIM rotation angle $\kappa$ has been filtered to reduce (random distributed, unbiased) noise. However, this only reduced the rms misfit in the CSC $N$ component by less than $1 \mathrm{nT}$, indicating that the noise is either not unbiased, independent distributed or that other, yet uninvestigated, effects contribute.

Figure 2 shows the data residuals for the Oersted(10c/99) model as a function of co-latitude. The largest residuals are found in the scalar field in the Northern polar cap (poleward of $80^{\circ}$ latitude or so). This is due to ionospheric currents in the (summer) polar cap. The effect will be discussed further in the next subsection. As a consequence, most of the rms misfit in the scalar field is due to these polar cap current systems; the misfit is much lower for dipole latitudes $\lambda_{\text {dip }}$ equatorwards of $50^{\circ}$, as can be seen from Table 1 . Introducing the weighting factor $w \propto \sin \vartheta$ (which was done to get an equal area coverage) of course minimizes the influence of polar cap currents on the model.

The three vector components show a systematic broadscale behavior. To guide the eye, fourth degree polynomials were fitted to the residuals and are shown with solid lines. The residuals indicate a depression of $B_{r}$ (of a few nT) at the equator. At the time of model submission and validation (November 1999) the reason for these residuals were unknown. However, in January 2000 we found that a slightly different model parameterization is able to reduce the observed broad-scale features: inclusion of an external coefficient $q_{2}^{0}$ in Eq. (2) removes some of the broad-scale features in $B_{r}$ and $B_{\vartheta}$; the coefficient is estimated to $q_{2}^{0}=$ $+2.2 \mathrm{nT}$. However, although reduced in amplitude, there are still broad-scale features even after the inclusion of this coefficient.

A new data set, consisting of about 8600 scalar data points and about 5200 vector triplets of geomagnetic quiet periods during December 18 to 30, became available after the IGRF submission. This dataset was analyzed and a model, called Oersted(01/00), was estimated using the same model parameterization as described above ( $q_{2}^{0}$ included, epoch 1999.98). Surprisingly there were no broad-scale features in the residuals, and the absolute values of the residual means do not exceed $0.3 \mathrm{nT}$ in contrast to the $2 \mathrm{nT}$ for $B_{r}$ of the Oersted(10c/99) model. The "Ørsted Initial Field Model" (Olsen et al., 2000), which is based on fully calibrated data (using the Euler angles of the Oersted(01/00) model) of geomagnetic 


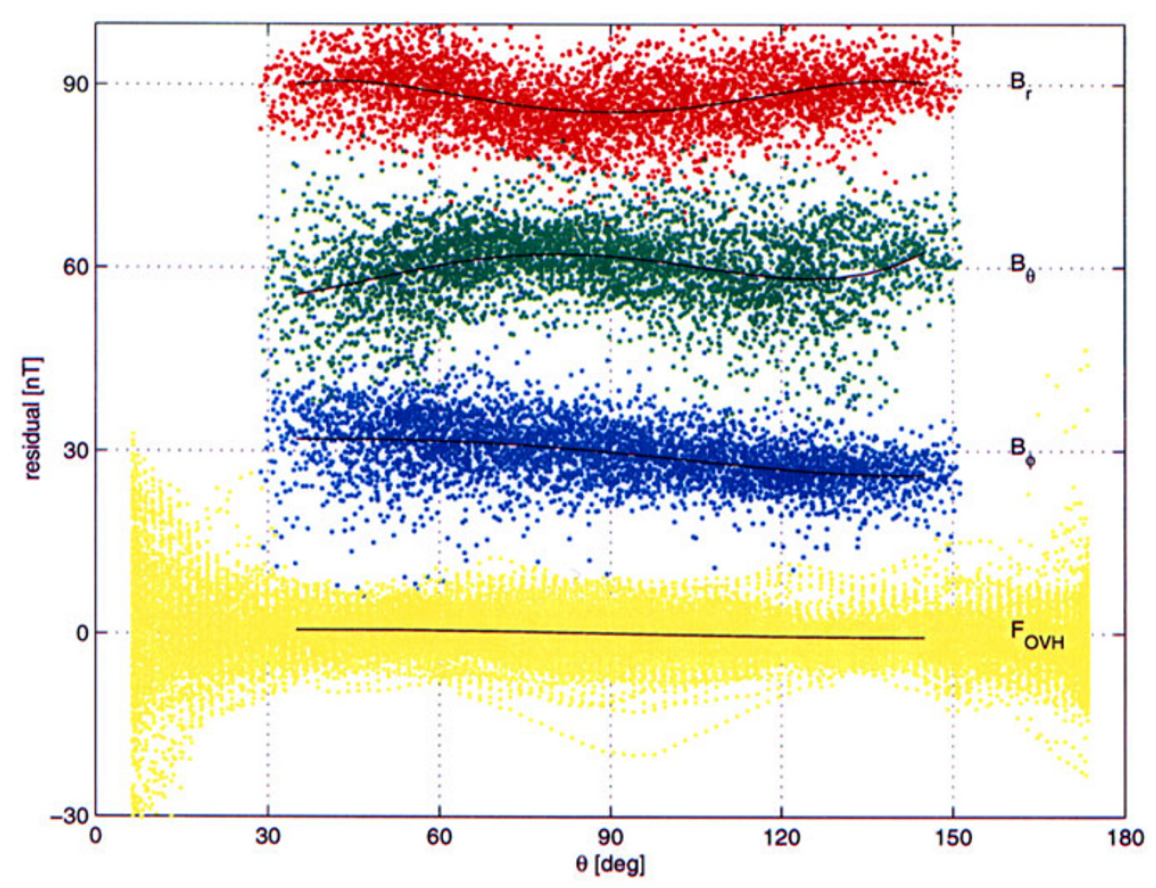

Fig. 2. Data residuals of the Oersted(10c/99) model as a function of co-latitude. The solid lines represent a fourth degree polynomial fit.
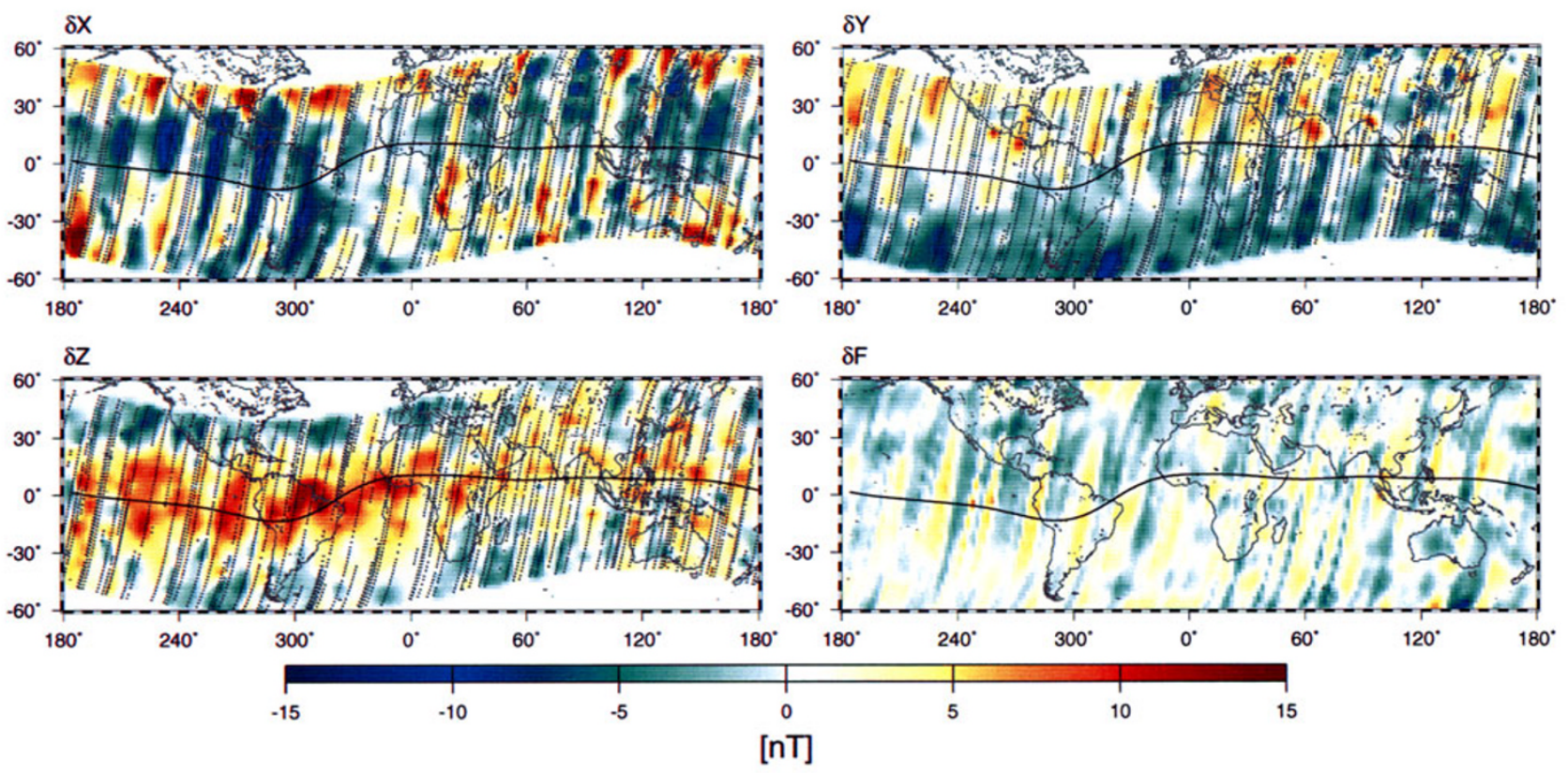

Fig. 3. Maps of the residuals of the Oersted(10c/99) model.

quiet periods between December 18, 1999 and January 21, 2000 confirms this data improvement. It is therefore likely that the broad scale features of the Oersted(10c/99) model are due satellite problems during the first months of the mission, resulting in biased vector data.

Figure 3 presents maps of the residuals of the Oersted(10c/99) model. An interpolation/smoothing using a nearest-neighbor algorithm has been used to produce these maps. These maps confirm the broad-scale features found before, and show that there is no longitudinal dependency of the residuals.

The misfit of the Oersted(10c/99) model (5.2 nT rms for the scalar data spanning six months and $5.9 \mathrm{nT}$ for the vector data spanning four months) indicate that the data do not contradict the applied secular variation model. However, more recent Ørsted data show that the secular variation probably is slightly different from the values given by the IGRF 95 SV model. The left part of Fig. 4 shows the coefficient $g_{1}^{0}$ for the four models (Oersted(10a/99), Oersted(10b/99), Oersted(10c/99) and Oersted(01/00)). The solid line (through 

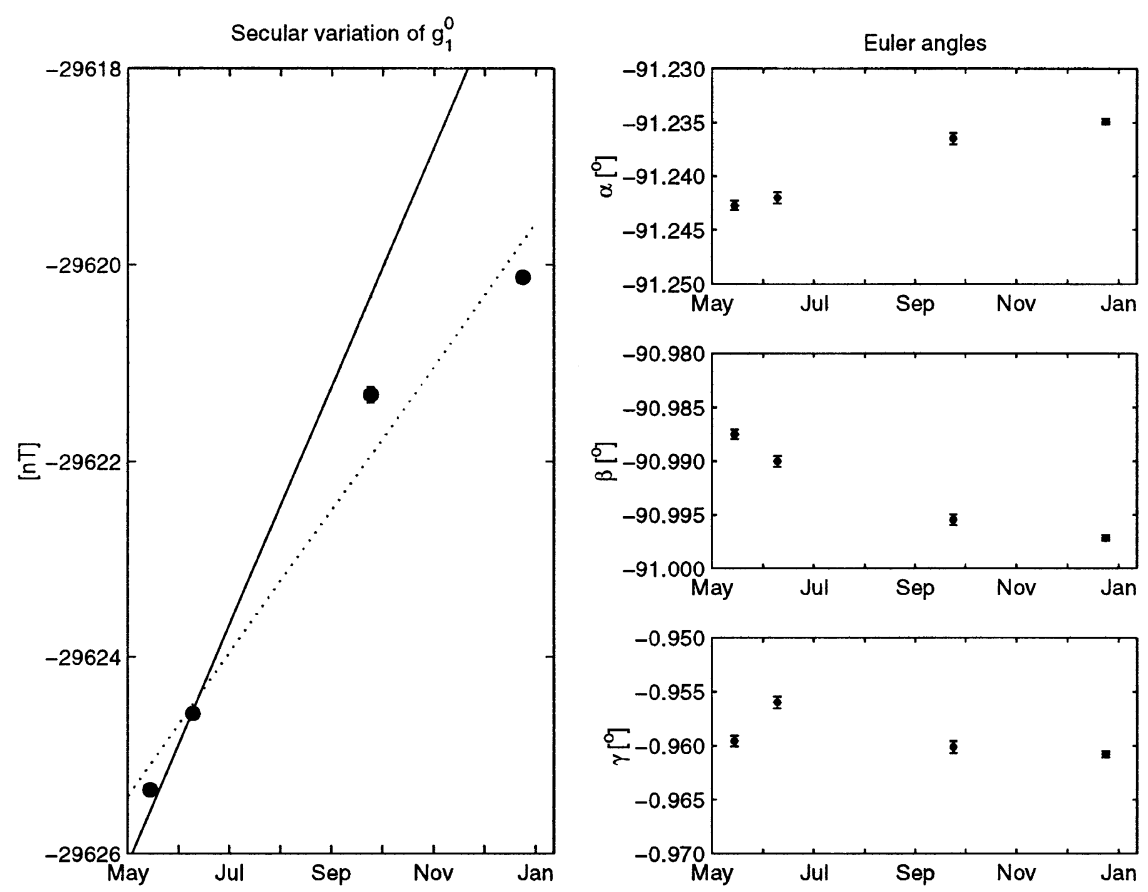

Fig. 4. Left: Expansion coefficient $g_{1}^{0}$ of the four models described in the text. The solid line is the time dependence $\dot{g}_{1}^{0}=14.6 \mathrm{nT} / \mathrm{yr}$ given by the IGRF $95 \mathrm{SV}$ model, and the dotted line is a least-squares fit to the $g_{1}^{0}$ coefficients of the three models obtained from the May, September and December data, respectively. Right: The Euler angles $\alpha, \beta$ and $\gamma$ describing the CSC-SIM rotation as found from the different data sets.

the value of the Oersted(10c/99) model) represents the time derivative $\dot{g}_{1}^{0}=14.6 \mathrm{nT} / \mathrm{yr}$ as given by the IGRF $95 \mathrm{SV}$ model, and the dotted line is a least-squares fit to the $g_{1}^{0}$ coefficients obtained from the May, September and December data (that means the Oersted(10a/99), Oersted(10b/99) and Oersted(01/00) model). This line corresponds to a time derivative of only $\dot{g}_{1}^{0}=9.0 \mathrm{nT} / \mathrm{yr}$. This discrepancy might be due to a change of the secular variation (the IGRF $95 \mathrm{SV}$ model is based on data gathered before 1995), due to the annual variability of external current systems (not accounted for when deriving the Ørsted models) or due to other, yet unexplored reasons.

The right part of Fig. 4 shows the values of the three Euler angles $\alpha, \beta$ and $\gamma$ for the different models. Although the differences between the models are considerably larger than the errorbars we do not believe that a real change of the Euler angles has occurred. The values obtained with the September and December data, respectively, differ by less than 7 arcseconds. This may indicate that the vector data of May are not of the same quality as the measurements taken during the last months of 1999. $\alpha$ and $\beta$ of the Oersted(10c/99) model are between the values obtained with the May and September data, but $\gamma$ is larger by 15 arcseconds. We believe that the Euler angles obtained from the December data set are the most reliable estimates. However, using slightly different values for deriving the other models probably accounts for no more than about $5 \mathrm{nT}$.

The lower panels of Fig. 5 show the model differences in $Z$. There is clear indication for a Backus effect (Stern and Bredekamp, 1975) since the largest discrepancies are found close to the dip-equator. We think that this is due to the coarse coverage of the vector data in the May and September model, respectively (cf. Fig. 1). However, this is mostly due to the expansion coefficients above $n=10$. The difference between the Oersted(10a/99) and the Oersted(10c/99) model for degree/order 1-10 is below $10 \mathrm{nT}$.

The top panel of the Figure presents the differences in the total intensity. The largest differences occur in the Oersted(10b/99)-Oersted(10c/99) difference at the magnetic pole, which is probably due to the contamination of the Oersted(10b/99) model by ionospheric polar cap currents.

\subsection{External contributions}

The values of the coefficients of the Oersted(10c/99) model describing external sources (and their Earth-induced counterparts) are presented in Table 2. The static external terms are rather well aligned with the dipole axis (pole position from external coefficients: $13^{\circ} \mathrm{N}, 60^{\circ} \mathrm{W}$ ). Amplitude of the external static term is $20.1 \mathrm{nT}$ in good agreement with the Magsat GSFC(12/83) model with $18.7 \mathrm{nT}$ (Langel and Estes, 1985). However, the Dst dependent coefficients are somewhat smaller than those found with Magsat $(-0.3$ compared to -0.6$)$. The reason is probably the much smaller number of data used for the Ørsted model. The ratio $g_{1, D s t}^{0} / q_{1, D s t}^{0}=$ $0.13 / 0.32=0.41$ is a reasonable value for induction with periods of a few days (Magsat: 0.27). As described in Section 2, the data have been selected according to the geomagnetic index $K p$ only, ignoring the value of the Dst index. Dst was generally between -20 and $+20 \mathrm{nT}$, with the exception of the first hours of September 23 for which Dst increases from $-100 \mathrm{nT}$ to $-40 \mathrm{nT}$.

Table 1 shows that the rms misfit of the scalar field is lower for the Oersted(10b/99) model compared to the Oersted(10a/99) model which is due to the much lower scatter of the Oersted(10b/99) residuals in the northern polar cap. 

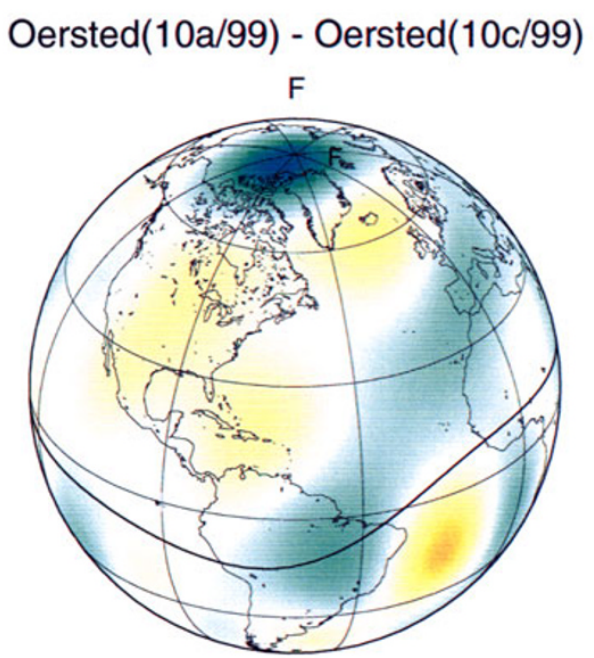

Z

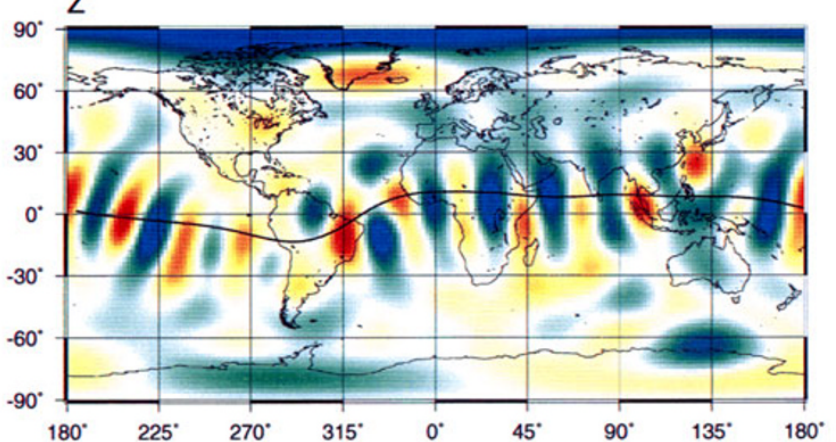

Oersted(10b/99) - Oersted(10c/99)

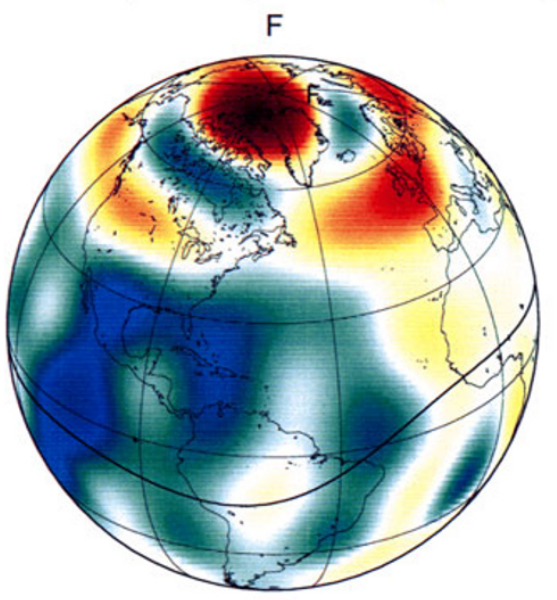

z

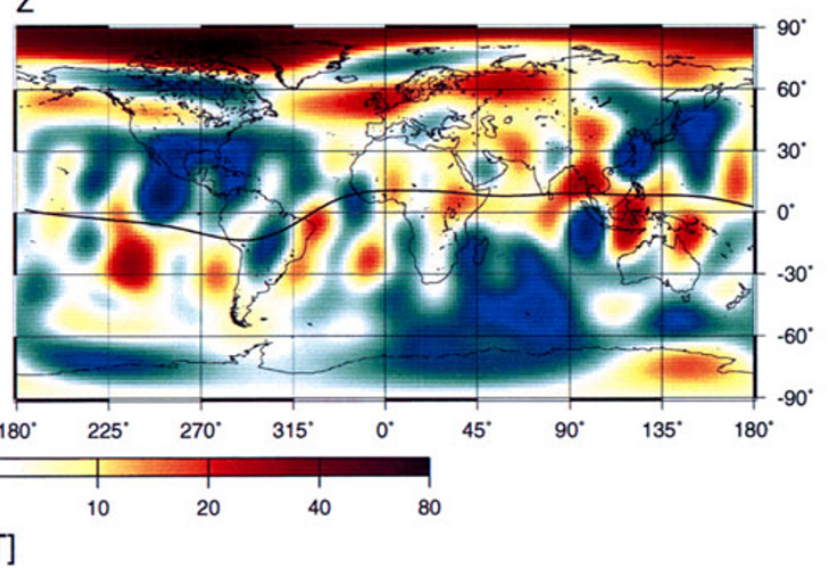

Fig. 5. Model differences for the $F$ component (top) and $Z$ component (bottom).

Table 2. External expansion coefficients of the Oersted(10c/99) model and the coefficients describing the Dst dependence.

\begin{tabular}{rrc}
\hline & \multicolumn{1}{c}{ Static } & Dst dependence \\
\hline$q_{1}^{0}$ & $19.55 \mathrm{nT}$ & -0.32 \\
$q_{1}^{1}$ & $2.29 \mathrm{nT}$ & -0.12 \\
$s_{1}^{1}$ & $-3.98 \mathrm{nT}$ & 0.12 \\
$g_{1}^{0}$ & $-29614.72 \mathrm{nT}$ & -0.13 \\
\hline
\end{tabular}

This is probably because the May-model is based on data during "away" as well as "toward" sector structure of the Interplanetary Magnetic Field (IMF), resulting in ionospheric polar cap currents of alternating sign. In contrast, the $y$ component of the IMF was always negative ( -2 to $-3 \mathrm{nT})$ for September 23-25. The corresponding ionospheric polar cap currents produce a positive vertical component in the northern polar cap of $50 \mathrm{nT}$ or more. This can be seen at the geomagnetic observatory Thule where the vertical component for September 23-25 is 50-100 nT above the mean. Ørsted observed an additional magnetic field of similar magnitude, which is the reason why the residuals in $Z$ and $F$ in the northern polar cap of Fig. 5 are almost $+80 \mathrm{nT}$ near the magnetic pole. Although there is less scatter in the northern polar cap in the September data, the Oersted(10b/99) model derived from these data is probably biased near the magnetic north pole due to the preferred direction of the ionospheric polar cap currents. Hence their contribution does not average out, as it is probably the case for the May data.

Figure 6 illustrates this further. The left panel shows the residuals of the May data (top) and September data (bottom) with respect to the model derived from the respective data. The scatter of the May data with respect to the Oersted $(10 \mathrm{a} / 99)$ model is much larger $(8.8 \mathrm{nT} \mathrm{rms})$ than the scatter of the September data with respect to the Oersted(10b/99) model (3.3 nT rms). However, when compared to the Oersted(10c/99) model (right panel) it becomes clear that the September data contain a contribution which is not described by the Oersted(10c/99) model. This additional signal is due to the polar cap currents which were included in the Oersted(10b/99) model.

\section{Conclusion}

Both the Oersted(10a/99) model and the Oersted(10b/99) model are probably contaminated by the Backus effect, as a result of the gaps in the global coverage with vector data near the dip-equator. This, however, mostly influences coefficients above degree and order 10. 

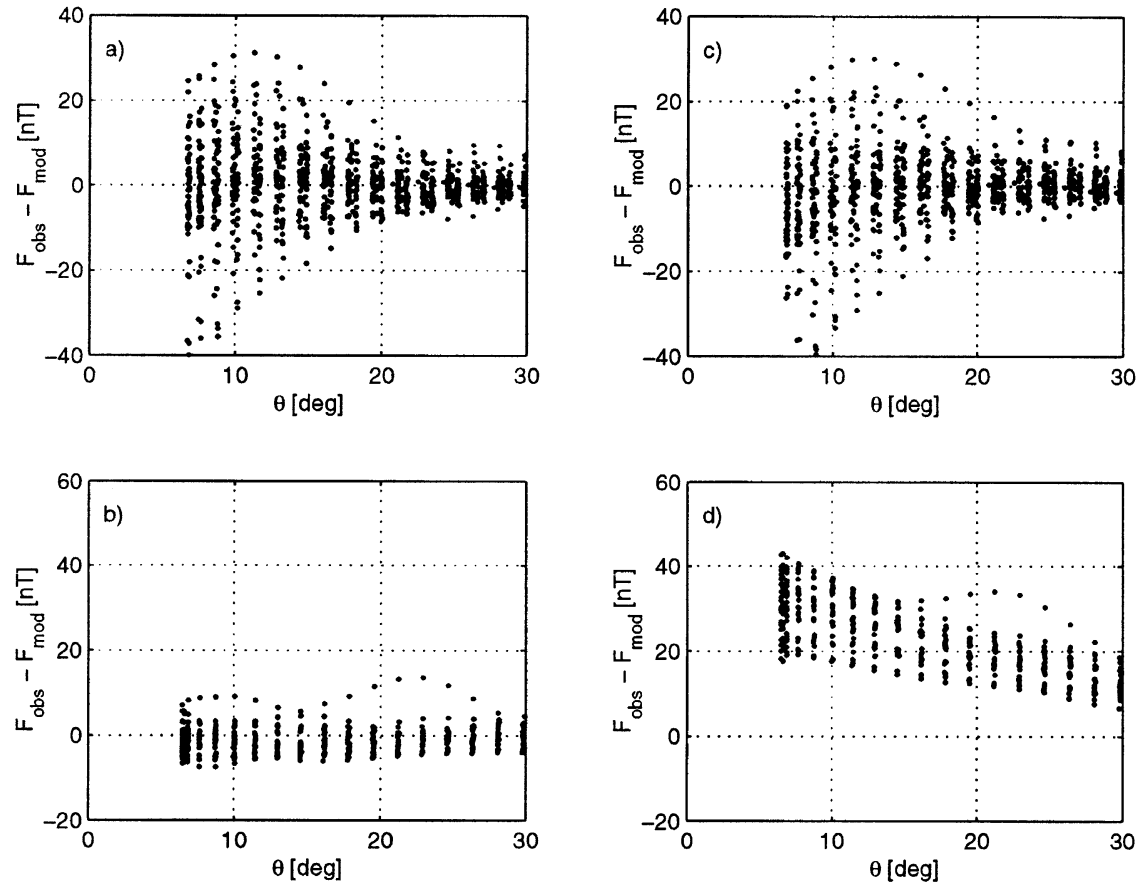

Fig. 6. The influence of ionospheric polar cap currents on field models. Residuals in $F$ as a function of co-latitude $\theta$ for a) May data minus Oersted(10a/99) model values, b) September data minus Oersted(10b/99) model values, c) May data minus Oersted(10c/99) model values, d) September data minus Oersted(10c/99) model values. Note the bias in case d).

In addition, the Oersted(10b/99) model is probably biased in the northern polar cap due to ionospheric polar cap currents with a preferred direction during September 23-25.

The Oersted(10c/99) model is based on scalar data spanning six months and vector data spanning four months. Global data coverage is very good. The model has been derived using the IGRF 95 SV model, and the statistics show that this SV model is in good agreement with the Ørsted data (OVH misfit: $5.2 \mathrm{nT}$ rms, CSC misfit: $5.9 \mathrm{nT}$ rms). However, analyses of more recent data indicate some discrepancies between the time change as observed by the satellite and that predicted by the SV model. Despite the broad-scale features in the residuals of the Oersted(10c/99) model and the deficiencies which have been found we believe that this model fully satisfies IGRF standards.

Acknowledgments. The Ørsted Project was made possible by extensive support from the Ministry of Trade and Industry, the Min- istry of Research and Information Technology and the Ministry of Transport in Denmark. Additional international and crucial support was provided from NASA, ESA, CNES and DARA.

\section{References}

Barton, C. E., International Geomagnetic Reference Field: the seventh generation, J. Geomag. Geoelectr., 49, 123-148, 1997.

Langel, R. A. and R. H. Estes, The near-Earth magnetic field at 1980 determined from MAGSAT data, J. Geophys. Res., 90, 2495-2510, 1985.

Olsen, N., et al., Ørsted initial field model, Geophys. Res. Lett., 27(22), 3607-3610, 2000.

Olsen, N., T. Risbo, P. Brauer, J. M. G. Merayo, F. Primdahl, and T. Sabaka, In-flight calibration methods used for the Ørsted mission, in Ground and In-Flight Space Magnetometer Calibration Techniques, edited by A. Balogh and F. Primdahl, ESA SP-490, ESA Publishing Division, ESTEC, Katwijk, The Netherlands, 2001.

Stern, D. P. and J. H. Bredekamp, Error enhancement in geomagnetic models derived from scalar data, J. Geophys. Res., 80, 1776-1782, 1975.

N. Olsen (e-mail: nio@dsri.dk), T. J. Sabaka, and L. Tøffner-Clausen 